\title{
Erratum
}

\section{Erratum: Heber et al., "Mice with Combined Gene Knock-Outs Reveal Essential and Partially Redundant Functions of Amyloid Precursor Protein Family Members"}

In the article "Mice with Combined Gene Knock-Outs Reveal Essential and Partially Redundant Functions of Amyloid Precursor Protein Family Members,” by Sabine Heber, Jochen Herms, Vladan Gajic, Johannes Hainfellner, Adriano Aguzzi, Thomas Rülicke, Hans Kretzschmar, Cornelia von Koch, Sangram Sisodia, Phillippe Tremml, Hans-Peter Lipp, David P. Wolfer, and Ulrike Müller, which appeared on pages 7951-7963 of the November 1, 2000 issue, the authors would like to address the following error in Figure 4. Parts of panels $a$ and $c$, depicting Nissl and synaptophysin stainings, were inadvertently duplicated. The authors regret this error. MAPII and TUNEL stainings (with nuclear fast counterstain to visualize nuclei) of panels $a$ and $c$ are correct. The microscopic slides date back to the year 2000 and were destroyed 2 years ago in 2018. This prevents the authors from providing new images of this dataset. This error does not affect the conclusions of Figure 4 that the lack of APLP2 in combination with a deficiency for either APP or APLP1, respectively, has no obvious effect on cortical architecture in newborn mice.

DOI: 10.1523/JNEUROSCI.1801-20.2020

\section{Erratum: Valenza et al., "Dysfunction of the Cholesterol Biosynthetic Pathway in Huntington's Disease"}

In the article "Dysfunction of the Cholesterol Biosynthetic Pathway in Huntington's Disease," by Marta Valenza, Dorotea Rigamonti, Donato Goffredo, Chiara Zuccato, Simone Fenu, Laure Jamot, Andrew Strand, Alessia Tarditi, Ben Woodman, Marco Racchi, Caterina Mariotti, Stefano Di Donato, Alberto Corsini, Gillian Bates, Rebecca Pruss, James M. Olson, Simonetta Sipione, Marzia Tartari, and Elena Cattaneo, which appeared on pages 9932-9939 of the October 26, 2005 issue, the authors report that in composing Figure $5 b$, the white panels in the membrane and nuclear fractions, indicating, respectively, the absence of nuclear and membrane protein contaminants, were duplicated by mistake. Examination of the original films shows similarly white panels indicating the absence of contaminating proteins in the two fractions. This error does not affect the conclusions of the article. The authors regret this error. 